\title{
Controlling the likelihood of rogue waves in an optically injected semiconductor laser via direct current modulation
}

\author{
S. Perrone, ${ }^{1}$ R. Vilaseca, ${ }^{1}$ J. Zamora-Munt, ${ }^{2}$ and C. Masoller ${ }^{1}$ \\ ${ }^{1}$ Departament de Física i Enginyeria Nuclear, Universitat Politécnica de Catalunya, Campus de Terrassa, Edif. GAIA, \\ Rambla de Sant Nebridi 21,08222 Terrassa, Barcelona, Spain \\ ${ }^{2}$ Instituto de Física Interdisciplinar y Sistemas Complejos, IFISC (CSIC-UIB), E-07122 Palma de Mallorca, Spain
}

(Received 5 February 2014; published 6 March 2014)

\begin{abstract}
Extreme and rare events are nowadays the object of intensive research. Rogue waves are extreme waves that appear suddenly in many natural systems, even in apparently calm situations. Here we study numerically the rogue wave dynamics in an optically injected semiconductor laser with external periodic forcing that is implemented via direct modulation of the laser pump current. In the region of optical injection parameters where the laser intensity is chaotic and occasional ultrahigh pulses occur, our aim is to control the system by applying a weak modulation. We find that for an adequate range of frequency and amplitude parameters, the modulation can completely suppress the extreme pulses. We also show that the interplay between modulation and an external source of noise can significantly modify their probability of occurrence. These results can motivate a range of experimental and theoretical investigations in other natural systems.
\end{abstract}

DOI: 10.1103/PhysRevA.89.033804

PACS number(s): 42.55.Px, 42.60.Mi, 05.45.-a, 42.65.Tg

\section{INTRODUCTION}

Extreme, rare, and devastating events such as tsunamis, market crashes, earthquakes, population extinctions, etc. are nowadays receiving a great deal of attention. A major challenge is to predict them and, if possible, to control them, making safer environments and systems. In oceanography, ultrahigh waves, referred to as rogue waves (RWs), have been extensively studied [1-4], and they have also been observed in many other systems [5-8]. Rogue waves, as opposed to tsunamis or solitons, can disappear in a short spatial length and not necessarily propagate for large distances.

Laboratory controlled experiments have allowed one to understand hydrodynamic RWs in terms of solutions of the nonlinear Schrödinger equation (NLSE) [9,10]. A variety of these solutions have been used to explain RWs in different systems. However, the need of a better and more accurate understanding of extreme phenomena has stimulated the research on families of solutions in systems of coupled wave equations such as the vector NLSE [11], coupled GrossPitaevskii equations [12], or Hirota systems [13]. In this context, several waves resonant interaction $[14,15]$ has been proposed as a mechanism of generation of RWs highlighting the existence of preferred length or time scales in the system [16].

In an optical system RWs were first reported by Solli et al. [17] using a microstructured optical fiber, in which extremely broadband radiation was generated from a narrow-band input. Since then, RWs have been observed in many optical systems (see [18] for a recent review). In vertical cavity surface emitting lasers, Ti:sapphire lasers, Raman lasers, and mode-locked lasers, rogue waves in the form of extreme intensity pulses, with a probability higher than that predicted by Gaussian statistics, have been observed experimentally and understood in terms of laser model simulations [19-26].

Since in nonlinear systems the chaotic behavior can often be suppressed by a weak periodic modulation of a control parameter [27-31], a main objective of our work is to investigate if rogue waves can also be controlled via a small periodic perturbation. Another relevant open question is the influence of noise. Since the interplay between noise and modulation can lead, in nonlinear systems, to stochastic resonance phenomena [32], another goal of our work is to analyze the interplay of noise and modulation in the statistics of the RW events.

With these purposes we study numerically the generation of RWs in an optically injected semiconductor laser with a periodic external forcing via a direct modulation of the laser pump current. We use the rate-equation model previously studied in $[20,26]$. In this system, without modulation deterministic RWs were found in simulations of noiseless rate equations [20]. We show here that for an adequate range of frequency and amplitude parameters, current modulation can completely suppress the RWs. We also show that the interplay between noise and modulation can significantly modify the likelihood of RWs, since depending on the modulation frequency and noise strength RWs can be either enhanced or diminished. By shedding light into the role of periodic forcing in a laser system that generates RWs, our findings can motivate experimental and theoretical investigations in other natural systems.

\section{MODEL}

The dynamics induced in a cw optically injected singlemode laser can be reproduced by a set of two rate equations for the slow envelope of the complex electric field $E$ and the carrier density $N$ as $[33,34]$

$$
\begin{gathered}
\frac{d E}{d t}=\kappa(1+i \alpha)(N-1) E+i \Delta \omega E \\
+\sqrt{P_{\mathrm{inj}}}+\sqrt{D} \xi(t), \\
\frac{d N}{d t}=\gamma_{N}\left[\mu(t)-N-|E|^{2}\right],
\end{gathered}
$$

where $\kappa$ is the field decay rate, $\alpha$ is the linewidth enhancement factor, $\gamma_{N}$ is the carrier decay rate, and $\mu(t)$ is the timedependent injection current parameter (normalized such that 
the threshold current for the solitary laser for constant $\mu$ is $\left.\mu_{\text {th }}=1\right) . \Delta v=\Delta \omega / 2 \pi$ is the optical frequency detuning and $P_{\text {inj }}$ is the optical injection strength. $\xi(t)$ is a complex Gaussian white noise of strength $D$ representing optical noise, due to spontaneous emission fluctuations, or due to incoherent external optical injection. In the next sections we will consider a sinusoidal current modulation, $\mu(t)=\mu_{0}+\mu_{\bmod } \sin \left(\omega_{\bmod } t\right)$, where $\mu_{0}$ is the dc bias current, $\mu_{\bmod }$ is the modulation amplitude, and $f_{\bmod }=\omega_{\bmod } / 2 \pi$ is the modulation frequency.

The model equations were numerically solved using the same parameters as in [20,26]: $\kappa=300 \mathrm{~ns}^{-1}, \alpha=3, \gamma_{N}=$ $1 \mathrm{~ns}^{-1}, P_{\mathrm{inj}}=60 \mathrm{~ns}^{-2}$, and the other parameters were varied. Time traces of $10 \mu \mathrm{sec}$ of the laser intensity, $I=\left|E^{2}\right|$, generated from random initial conditions have been simulated.

As RWs are ultrahigh pulses when compared with the average height of all the pulses, they are characteristic of the tail of "L-shaped" probability density functions (PDFs). Thus a suitable method to detect them is based on the analysis of the first- and second-order moments of the PDF of the intensity pulse heights. Following $[20,26]$ we consider that a pulse is a RW if its height is higher than a threshold, $\tau$, that is a function of the mean value of the intensity, $\langle I\rangle$, and its standard deviation, $\sigma_{I}$. Here we use $\tau=\langle I\rangle+6 \sigma_{I}$. Similar results were obtained with a higher threshold $\left(8 \sigma_{I}\right.$ instead of $\left.6 \sigma_{I}\right)$ even though the number of detected RWs was considerably smaller. In order to properly choose the threshold we have taken into account that too high thresholds have the drawback of detecting a small number of extreme pulses, requiring long simulation times, while too small thresholds could detect pulses that do not belong to the tail of the distribution of pulse heights. We found that the use of $6 \sigma_{I}$ was an adequate compromise between these two facts.

\section{RESULTS}

\section{A. Influence of optical noise in the occurrence of rogue waves}

We begin by discussing the role of optical noise in the occurrence of RWs in the injected laser, without current modulation, i.e., $\mu=\mu_{0}$. Zamora-Munt et al. [26] have shown that optical noise (representing spontaneous emission, or incoherent optical injection) can be employed either to enhance or to suppress the probability of RW occurrence. Our analysis confirmed this observation but also revealed certain parameter regions where the influence of noise depends on the noise strength: while weak noise reduces the number of RWs, stronger noise significantly increases it.

This effect is shown in Fig. 1, which displays in color code the number of RWs in the parameter space $\left(\mu_{0}, \Delta v\right)$ for four different values of the noise strength. Note that the color code is in logarithmic scale, such that white regions correspond to zero detected RWs and dark regions (dark red online) correspond to several hundreds of detected RWs. Figure 1(a) shows the deterministic case $(D=0)$. The RWs detected under this condition will be referred to as deterministic optical RWs. With weak noise strength $\left(D=10^{-4} \mathrm{~ns}^{-1}\right.$ representing internal spontaneous emission) the number of detected RWs decreases, as shown in panel 1(b), in good agreement with the observations in [26]. Figures 1(c) and 1(d) present the results obtained with higher noise strength $\left(D=10^{-3} \mathrm{~ns}^{-1}\right.$
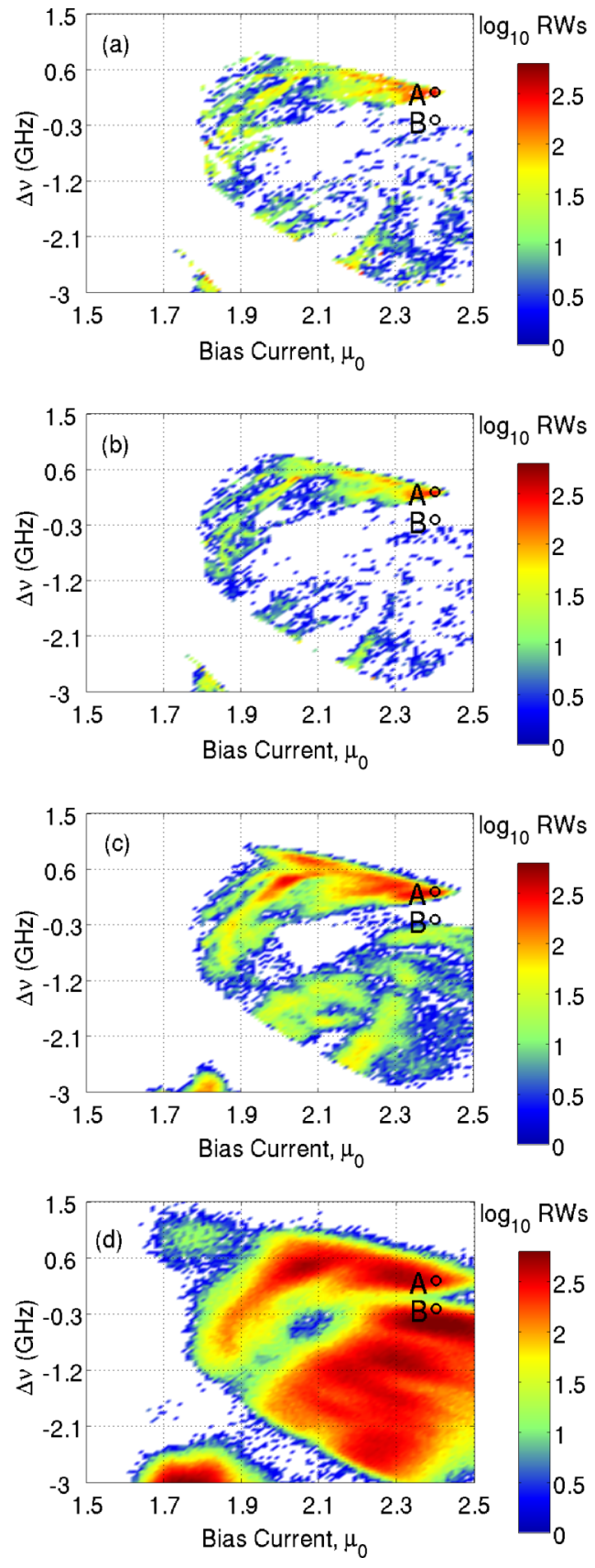

FIG. 1. (Color online) Number of detected RWs in the parameter space (pump current $\mu_{0}$; detuning $\Delta v$ ), when no current modulation is applied. The color code is plotted in logarithmic scale in order to increase the contrast of the regions with a small number of RWs. In the white regions no RWs are detected. The noise strength is $D=0$ (a), $10^{-4} \mathrm{~ns}^{-1}$ (b), $10^{-3} \mathrm{~ns}^{-1}$ (c), and $10^{-2} \mathrm{~ns}^{-1}$ (d). The dots labeled as $A$ and $B$ indicate the parameters used in the next section: for point $A, \mu_{0}=2.4$ and $\Delta v=0.22 \mathrm{GHz}$; for point $B, \mu_{0}=2.4$ and $\Delta v=-0.24 \mathrm{GHz}$.

and $D=10^{-2} \mathrm{~ns}^{-1}$, representing external incoherent optical injection). It can be noticed that the dark regions (color online) are wider, revealing regions of purely noise-induced RWs, and also, in regions where deterministic RWs occur, it can be noticed that the number of RWs generally increases with respect to the deterministic situation. 

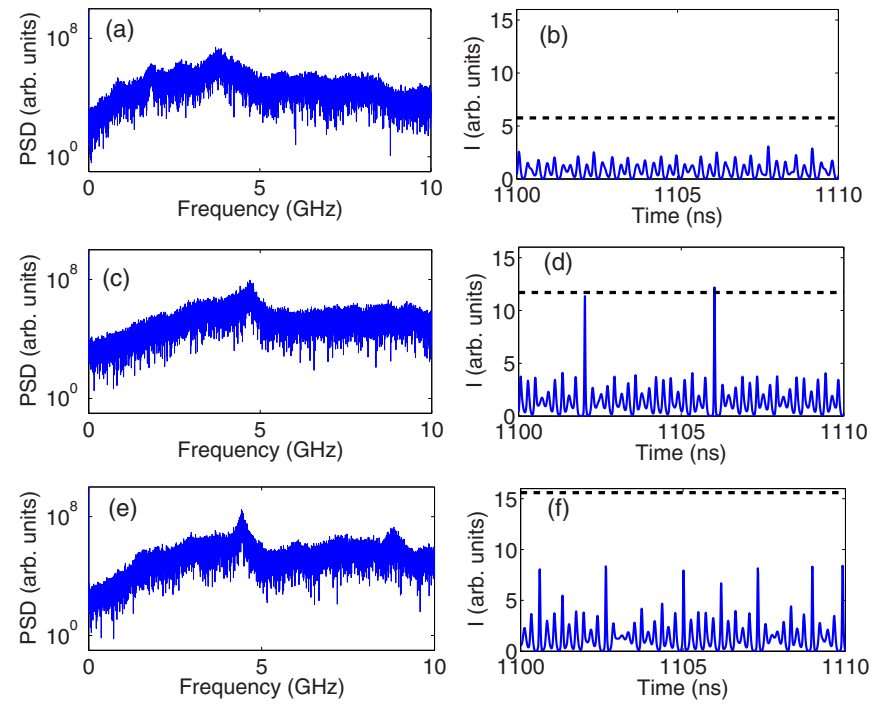

FIG. 2. (Color online) Power spectral density (a), (c), and (e) and intensity time trace (b), (d), and (f), when no current modulation is applied. The parameters $\mu_{0}=1.8$ and $\Delta v=0.22 \mathrm{GHz}$ (a), (b), $\mu_{0}=2.4$ and $\Delta v=0.22 \mathrm{GHz}(\mathrm{c}),(\mathrm{d})$ (these parameters are indicated with label $A$ in Fig. 1), and $\mu_{0}=2.4$ and $\Delta v=-0.24 \mathrm{GHz}$ (e), (f) (these parameters are indicated with label $B$ in Fig. 1). The horizontal line indicates the RW threshold. The noise strength is $D=10^{-4} \mathrm{~ns}^{-1}$.

In order to investigate the influence of periodic modulation, it is crucial to previously understand the characteristic time scales of the chaotic dynamics induced by optical injection. In the parameter region where RWs occur the main characteristic time scale is the laser relaxation oscillation frequency, which in the solitary laser increases linearly with $\sqrt{\mu-\mu_{\text {th }}}$, while in the optically injected laser it also depends on the injection parameters $[35,36]$.

Figure 2 presents typical examples of the power spectral density (PSD) and the corresponding intensity time trace, for two values of the laser bias current, $\mu_{0}=1.8$ and 2.4, and two values of the injection detuning, $\Delta v=0.22 \mathrm{GHz}$ and $-0.24 \mathrm{GHz}$. These parameters were chosen such that the intensity presents chaotic oscillations [20]. The PSD, Figs. 2(a), 2(c), and 2(e), displays a peak at a frequency close to the relaxation oscillation frequency of the solitary laser, $f_{\text {ro }}=$ $\sqrt{2 k(\mu-1) / \gamma_{N}} / 2 \pi$ (for $\mu_{0}=1.8, f_{\text {ro }}=3.5 \mathrm{GHz}$, while for $\mu_{0}=2.4, f_{\text {ro }}=4.5 \mathrm{GHz}$ ). For $\mu_{0}=1.8$ the intensity oscillations are of small amplitude and the probability to observe a RW is very small; see Fig. 2(b). For $\mu_{0}=2.4$, if $\Delta v=0.22 \mathrm{GHz}$ irregular oscillations dominate the dynamics but occasionally an ultrahigh pulse whose amplitude is above the RW threshold occurs; see Fig. 2(d). These parameters are indicated with label $A$ in Fig. 1, and have been chosen because for these parameters a high number of RWs were detected (about 300 in $10 \mu \mathrm{sec}$ ). With a negative detuning $(\Delta v=-0.24 \mathrm{GHz}$, indicated with label $B$ in Fig. 1) frequent high pulses occur, but they are not RWs as they are below the threshold; see Fig. 2(f).

Comparison between Figs. 2(a), 2(b), and 2(c) tells us that it is difficult to identify specific features in the PSD spectrum which could be unambiguously associated to the appearance of RWs (as the PSD does not provide information about the pulse heights). Nevertheless, from these PSDs we can infer that the relevant frequency interval to modulate the system (see below) is $0-5 \mathrm{GHz}$, as the modulation response of a semiconductor laser is limited by the relaxation oscillation frequency [34].

\section{B. RW suppression by weak current modulation}

In this section we adopt the parameter settings corresponding to points $A$ and $B$ in Fig. 1, and apply a sinusoidal modulation of amplitude $\mu_{\text {mod }}$ and frequency $f_{\text {mod }}$. For point $A$ (for which RWs appear in the absence of modulation), the number of detected RWs as a function of the modulation frequency, $f_{\text {mod }}$, and the modulation amplitude (normalized to the dc value, $\mu_{0}$ ), is displayed in Fig. 3 for three levels of noise, $D=0,10^{-4} \mathrm{~ns}^{-1}$, and $10^{-3} \mathrm{~ns}^{-1}$. As in the previous section, we note here that the color code is in logarithmic scale, such that white regions correspond to zero detected RWs and the dark regions (dark red online) correspond to several hundreds of detected RWs.

In Fig. 3(a) one can notice that the addition of weak current modulation leads to a substantial reduction of the number
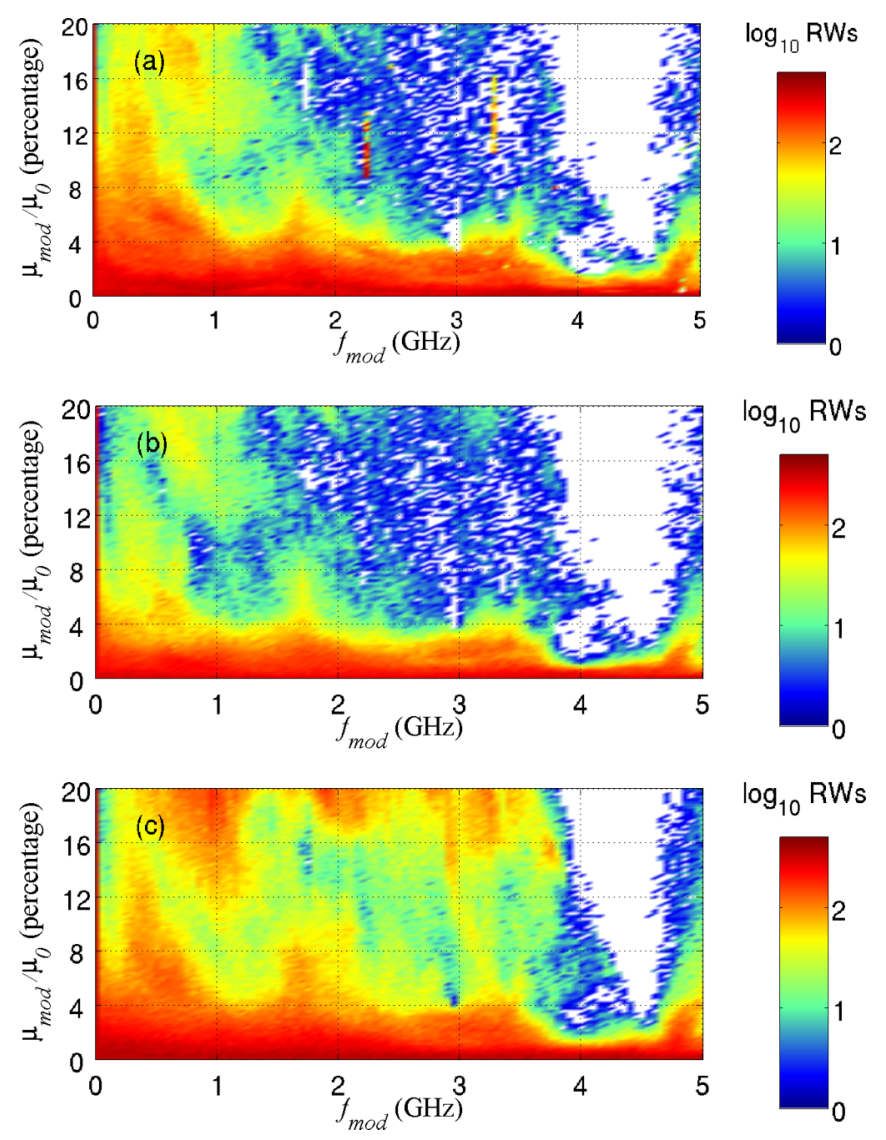

FIG. 3. (Color online) Number of detected RWs in the parameter space (modulation frequency $f_{\text {mod }}$; normalized modulation amplitude $\left.\mu_{\text {mod }} / \mu_{0}\right)$. The color code is plotted in logarithmic scale in order to increase the contrast of the regions with a small number of RWs. In the white regions no RWs are detected. The parameters are such that the intensity dynamics displays deterministic RWs in the absence of noise and modulation: $\mu_{0}=2.4$ and $\Delta v=0.22 \mathrm{GHz}$ [point labeled $A$ in Fig. 1(a)]. The noise strength is $D=0$ (a), $10^{-4} \mathrm{~ns}^{-1}$ (b), and $10^{-3} \mathrm{~ns}^{-1}(\mathrm{c})$. 
of deterministic RWs. Moreover, the RWs are completely suppressed for modulation frequencies close to $f_{\text {ro }}(4.5 \mathrm{GHz}$ for $\left.\mu_{0}=2.4\right)$. This effect is seen also when noise is included in the simulations; see Figs. 3(b) and 3(c). In addition, for weak modulation amplitude and zero or weak noise, at least two other modulation frequencies for which the number of RWs decreases can be seen in Fig. 3: one at about $1 \mathrm{GHz}$ and the other at about $2.5 \mathrm{GHz}$.

In Fig. 3 one can also observe that the number of detected RWs depends in a nonmonotonous way on the noise strength. Comparing with the deterministic case [Fig. 3(a)], noise of moderate strength leads to a global reduction of the number of RWs [Fig. 3(b)]. However, for stronger noise the number of detected RWs increases and the region of complete suppression (white region) shrinks [Figs. 3(c) and 3(d)]. Taken together, these results indicate that current modulation with frequency close to the resonance frequency of the laser can be combined with an optimal noise level in order to minimize the occurrence of RWs. Further increasing the noise level, beyond the values considered in Fig. 3, will also lead to a suppression of RWs, as in the limit of very high noise the dynamics will be fully dominated by the noise.

The suppression of RWs due to current modulation can be understood in the following terms: the modulation induces more frequent high (but not extremely high) pulses, which results in an increase of the RW threshold. The effect is more apparent in Fig. 4(f), which corresponds to modulation just at the relaxation oscillation frequency at $4.5 \mathrm{GHz}$ : the number of relatively large peaks in the time series increases, but at the same time the peaks take less extreme values (i.e., not so large, not so small) than in the other subfigures, so that no RWs appear [in agreement with Fig. 3(b)]. In Figs. 4(b),
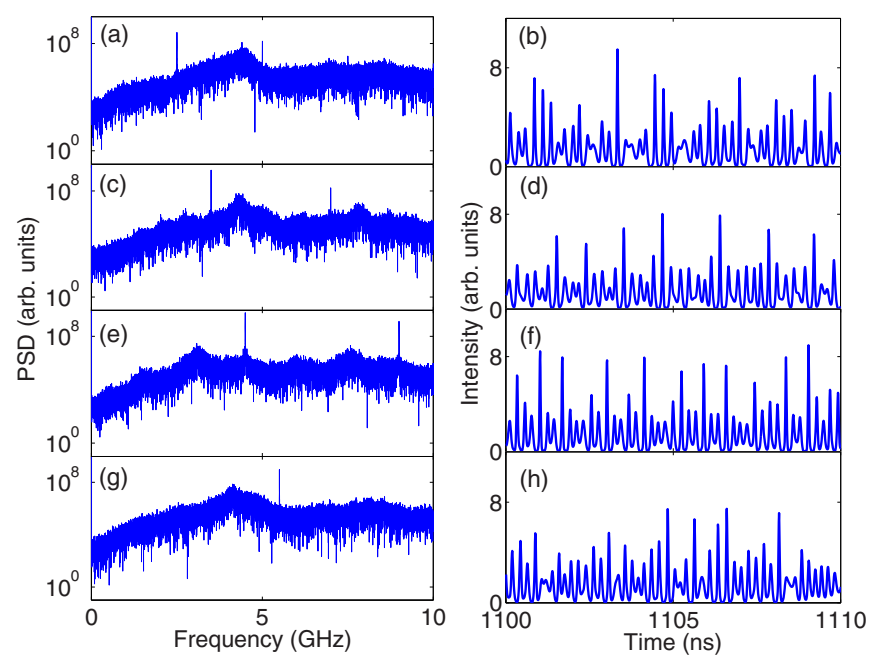

FIG. 4. (Color online) Influence of current modulation for parameters such that RWs occur without modulation (point labeled $A$ in Fig. 1 with $\Delta v=0.22 \mathrm{GHz}$ and $\mu_{0}=2.4$ ). Power spectral density (PSD, left column) and intensity time series (right column) when the modulation frequency is $f_{\text {mod }}=2.5 \mathrm{GHz}(\mathrm{a}),(\mathrm{b}), 3.5 \mathrm{GHz}$ (c), (d), $4.5 \mathrm{GHz}(\mathrm{e})$, (f), and $5.5 \mathrm{GHz}(\mathrm{g})$, (h). The modulation relative amplitude is $\mu_{\bmod } / \mu_{0}=0.16$ and the noise strength is $D=$ $10^{-4} \mathrm{~ns}^{-1}$. Without modulation the PSD and the intensity time series are displayed in Figs. 2(c) and 2(d). 4(d), and 4(h), however, a more pronounced modulation of the peaks (leading to both larger and smaller peaks) is apparent which might be due to the beating between the modulation and relaxation oscillation frequencies. In these cases some RWs appear, but in a number much smaller than in the case of the unmodulated system [see Fig. 3(b)]. These considerations are confirmed by the distribution of pulse heights included in Fig. 7 below [see left-column subfigures: Figs. 7(c), 7(e), 7(g), and 7(i) just correspond to the four cases shown in Fig. 4, and can be compared with Fig. 7(a) which corresponds to the unmodulated case]. Thus, with modulation the pulses of high amplitude become regular, and the risk of anomalous, ultrahigh pulses is significantly reduced or even completely suppressed. This indicates that adding an external weak periodic forcing is a suitable method to create small discharges that can prevent extreme phenomena by simply inducing them periodically on a smaller scale. Just to give a phenomenological description about the role of the modulation, we can think of controlled small avalanches in a snow-covered mountain in order to reduce the amount of accumulated snow that could feed a large and dangerous avalanche.

\section{Rogue waves induced by current modulation}

Let us now consider the influence of modulation for parameters where, without modulation, no RWs occur. We choose point $B$ in Fig. 1. The influence of the modulation parameters in the deterministic case $(D=0)$ is presented in Fig. 5(a), where one can observe that almost for all the range of modulation frequencies considered RWs are detected, except, as before, for modulation frequencies close to the laser relaxation oscillation frequency. However, the number of detected RWs is in general not very large; for relatively large modulation amplitudes and low noise [upper part in Figs. 5(a) and 5(b)] the number of RWs approaches, but remains below, the number found in point $A$ [compare with the upper part of Figs. 3(a) and 3(b)].

As in point $A$, in point $B$ including weak noise diminishes the number of RWs [Fig. 5(b)], an effect which is more clearly observed at low modulation frequencies. For example, notice in Fig. 5(b), that for $f_{\text {mod }} \approx 1 \mathrm{GHz}$, the number of RWs detected is smaller than in Fig. 5(a). With a stronger noise the RW regions expand, as shown in Fig. 5(c) and RWs are observed even without modulation $\left(\mu_{\text {mod }}=0\right)$.

Figure 6 displays the PSD and the intensity time series for the same modulation amplitude and frequencies as in Fig. 4, and one can see that indeed the effect of weak modulation in point $B$ is the same as in point $A$ : all comments made for Fig. 4 also apply to Fig. 6. To further confirm these observations, Fig. 7 displays the distribution of pulse heights in point $A$ (left column) and point $B$ (right column). The complete suppression of RWs is observed in panels $(\mathrm{g})$ and $(\mathrm{h})$, where $f_{\text {mod }} \approx f_{\text {ro }}$.

\section{CONCLUSIONS}

In this work we have investigated the dynamics of an optically injected semiconductor laser subjected to periodic forcing, which was implemented via a sinusoidal modulation of the laser bias current. The focus of the study was the generation of ultrahigh pulses, referred to as rogue waves (RWs), 

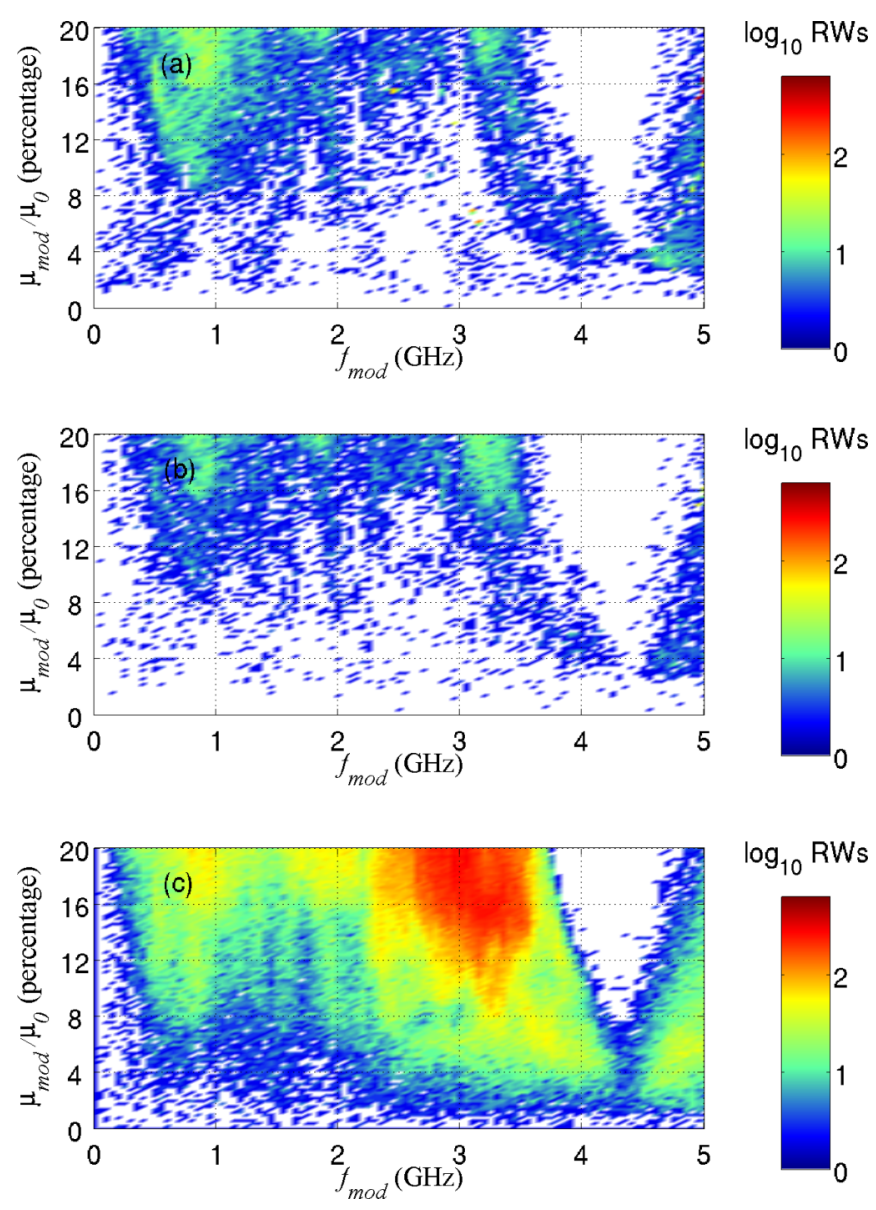

$\log _{10}$ RWs

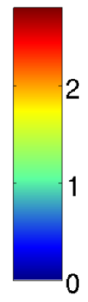

FIG. 5. (Color online) Number of detected RWs in the parameter space (modulation frequency $f_{\text {mod }}$; normalized modulation amplitude $\left.\mu_{\text {mod }} / \mu_{0}\right)$. The color code is plotted in logarithmic scale in order to increase the contrast of the regions with a small number of RWs. In the white regions no RWs are detected. The parameters are such that the intensity dynamics, in the absence of modulation, does not display RWs: $\mu_{0}=2.4$ and $\Delta v=-0.24 \mathrm{GHz}$ (point labeled $B$ in Fig. 1). The noise strength is $D=0$ (a), $10^{-4} \mathrm{~ns}^{-1}$ (b), and $10^{-3} \mathrm{~ns}^{-1}(\mathrm{c})$.

and specifically, the role of noise and modulation (and of the interplay between them) on the dynamics.

Concerning noise, its effect depends on the noise strength: while moderate noise strength decreases the number of RWs (in good agreement with a previous study [26]), stronger noise increases the likelihood of RWs and enhances the parameter region where they occur (see Fig. 1). Regarding the modulation, in parameter regions where there are deterministic RWs, the main effect of the modulation is to stabilize the irregular pulsing dynamics, and as the pulse heights become more regular the number of RWs decreases [see Fig. 3(a)]. On the other hand, in parameter regions where no deterministic RWs occur without modulation, with modulation a few RWs can be induced, in particular at definite frequency ranges below the relaxation oscillation frequency (see Fig. 5). For relatively large modulation amplitudes and low noise the number of RWs is in general rather insensitive to the behavior (presence or absence of RWs) of the unmodulated system.
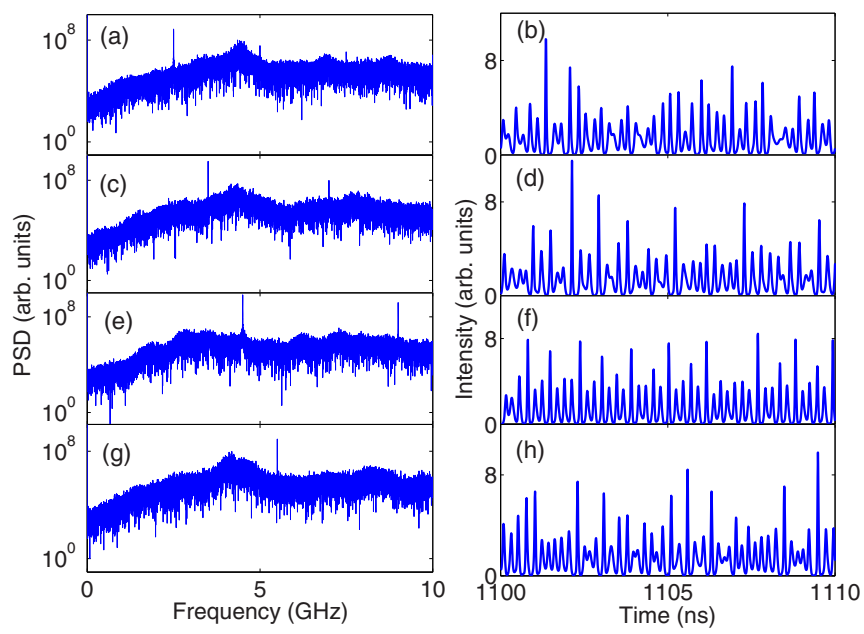

FIG. 6. (Color online) Influence of current modulation for parameters such that no RWs occur without modulation (point labeled $B$ in Fig. 1 with $\Delta v=-0.24 \mathrm{GHz}$ and $\mu_{0}=2.4$ ). Power spectral density (PSD, left column) and intensity time series (right column) when the modulation frequency is $f_{\text {mod }}=2.5 \mathrm{GHz}$ (a), (b), $3.5 \mathrm{GHz}$ (c), (d), $4.5 \mathrm{GHz}$ (e), (f), and $f_{\text {mod }}=5.5 \mathrm{GHz}(\mathrm{g})$, (h). The modulation relative amplitude is $\mu_{\text {mod }} / \mu_{0}=0.16$ and the noise strength is $D=10^{-4} \mathrm{~ns}^{-1}$. Without modulation the PSD and intensity time series is displayed in Figs. 2(e) and 2(f).

Regarding the interplay between noise and modulation, we found that noise of moderate strength can suppress or reduce the number of modulation-induced RWs [compare Figs. 5(a) and 5(b)]. At larger noise strengths, the tendency for noise to induce RWs (as it occurs in the unmodulated case) progressively shows up [compare Figs. 5(b) and $5(\mathrm{c})]$. In turn, modulation at frequencies close to the
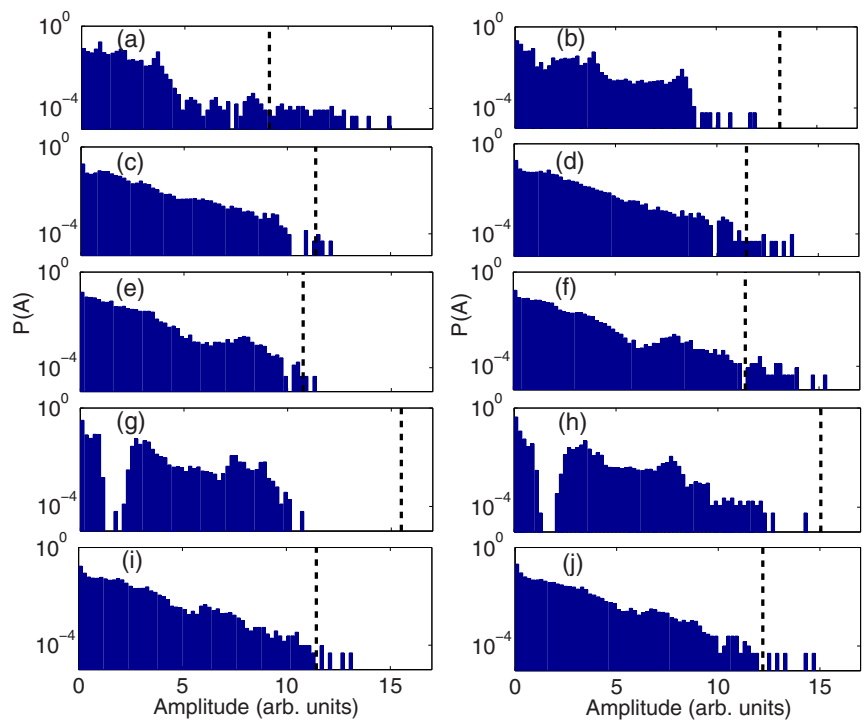

FIG. 7. (Color online) Pulse amplitude distribution in points $A$ (left column) and $B$ (right column). In panels (a), (b) no modulation is included; in (c)-(j) the parameters are as in Figs. 4 and 6. The vertical line indicates the RW threshold. The complete suppression of RWs is observed for a modulation frequency close to the relaxation oscillation frequency, in panels $(\mathrm{g})$ and $(\mathrm{h})$. 
characteristic time scale of the pulsing dynamics significantly diminishes and can even completely suppress noise-induced rogue waves, in a wide domain of modulation amplitudes [see white regions in Figs. 5(b) and 5(c) appearing for frequencies close to the relaxation oscillation frequency of the laser].

Summarizing, our results show that the occurrence of RWs can be controlled via a weak periodic forcing of appropriate modulation frequency and/or via incoherent optical injection (noise) of appropriate strength. As we have shown here that adding weak modulation results in an increased regularity of the amplitude of the pulses, it would be interesting as a future study to analyze the influence of current modulation in the periodicity of the pulsating dynamics, in particular, to analyze the effect of the modulation in the distribution of waiting times between two consecutive RWs. We hope that our findings will motivate not only new experiments in this laser system, but also, experimental and theoretical investigations in other natural systems.

\section{ACKNOWLEDGMENTS}

This research was supported in part by the Spanish Ministerio de Ciencia e Innovación through projects FIS2012-37655C02-01 and FIS2011-29734-C02-01, the Air Force Office Scientific Research through project FA-8655-10-1-3075, and by the Agència de Gestió d'Ajuts Universitaris i de Recerca (AGAUR), Generalitat de Catalunya, through project 2009 SGR 1168. C.M. also acknowledges the ICREA foundation for financial support. J.Z.M. acknowledges the support by Ministerio de Economia y Competitividad (Spain) under the INTENSE@COSYP project FIS2012-30634.
[1] B. S. White and B. Fornberg, J. Fluid Mech. 355, 113 (1998).

[2] M. Hopkin, Nature (London) 430, 492 (2004).

[3] S. Aberg and G. Lindgren, Probab. Eng. Mech. 23, 359 (2008).

[4] L. Cavaleri, L. Bertotti, L. Torrisi, E. Bitner-Gregersen, M. Serio, and M. Onorato, J. Geophys. Res. 117, C00J10 (2012).

[5] A. N. Ganshin, V. B. Efimov, G. V. Kolmakov, L. P. MezhovDeglin, and P. V. E. McClintock, Phys. Rev. Lett. 101, 065303 (2008).

[6] Y. V. Bludov, V. V. Konotop, and N. Akhmediev, Phys. Rev. A 80, 033610 (2009).

[7] Z. Y. Yan, Commun. Theor. Phys. 54, 947 (2010).

[8] M. Onorato, S. Residori, U. Bortolozzo, A. Montina, and F. T. Arecchi, Phys. Rep. 528, 47 (2013).

[9] A. Chabchoub, N. P. Hoffmann, and N. Akhmediev, Phys. Rev. Lett. 106, 204502 (2011).

[10] A. Chabchoub, N. Hoffmann, M. Onorato, A. Slunyaev, A. Sergeeva, E. Pelinovsky, and N. Akhmediev, Phys. Rev. E 86, 056601 (2012).

[11] F. Baronio, A. Degasperis, M. Conforti, and S. Wabnitz, Phys. Rev. Lett. 109, 044102 (2012).

[12] Y. V. Bludov, V. V. Konotop, and N. Akhmediev, Eur. Phys. J.: Spec. Top. 185, 169 (2010).

[13] S. Chen and L. Y. Song, Phys. Rev. E 87, 032910 (2013).

[14] F. Baronio, M. Conforti, A. Degasperis, and S. Lombardo, Phys. Rev. Lett. 111, 114101 (2013).

[15] S. Chen, P. Grelu, and J. M. Soto-Crespo, Phys. Rev. E 89, 011201(R) (2014).

[16] S. Barkhofen, J. J. Metzger, R. Fleischmann, U. Kuhl, and H.-J. Stöckmann, Phys. Rev. Lett. 111, 183902 (2013).

[17] D. R. Solli, C. Ropers, P. Koonath, and B. Jalali, Nature (London) 450, 1054 (2007).

[18] N. Akhmediev, J. M. Dudley, D. R. Solli, and S. K. Turitsyn, J. Opt. 15, 060201 (2013).

[19] M. Kovalsky, A. A. Hnilo, and J. R. Tredicce, Opt. Lett. 36, 4449 (2011).
[20] C. Bonatto, M. Feyereisen, S. Barland, M. Giudici, C. Masoller, Jose R. Rios Leite, and J. R. Tredicce, Phys. Rev. Lett. 107, 053901 (2011).

[21] A. N. Pisarchik, R. Jaimes-Reategui, R. Sevilla-Escoboza, G. Huerta-Cuellar, and M. Taki, Phys. Rev. Lett. 107, 274101 (2011)

[22] J. M. Soto-Crespo, P. Grelu, and N. Akhmediev, Phys. Rev. E 84, 016604 (2011).

[23] A. Zaviyalov, O. Egorov, R. Iliew, and F. Lederer, Phys. Rev. A 85, 013828 (2012).

[24] C. Lecaplain, P. Grelu, J. M. Soto-Crespo, and N. Akhmediev, Phys. Rev. Lett. 108, 233901 (2012).

[25] P. Grelu and N. Akhmediev, Nat. Photon. 6, 84 (2012).

[26] J. Zamora-Munt, B. Garbin, S. Barland, M. Giudici, Jose R. Rios Leite, C. Masoller, and J. R. Tredicce, Phys. Rev. A 87, 035802 (2013).

[27] A. Kul'minskii, R. Vilaseca, and R. Corbalán, Phys. Rev. E 61, 2500 (2000).

[28] R. Vilaseca, A. Kul'minskii, and R. Corbalán, Phys. Rev. E 54, 82 (1996).

[29] U. Feudel and C. Grebogi, Chaos 7, 597 (1997).

[30] A. N. Pisarchik, V. N. Chizhevsky, R. Corbalán, and R. Vilaseca, Phys. Rev. E 55, 2455 (1997).

[31] Handbook of Chaos Control, 2nd ed., edited by H. G. Schuster (Wiley-VCH Verlag GmbH \& Co. KGaA, Weinheim, Germany, 2008).

[32] L. Gammaitoni, P. Hanggi, P. Jung, and F. Marchesoni, Rev. Mod. Phys. 70, 223 (1998).

[33] S. Wieczorek, B. Krauskopf, T. B. Simpson, and D. Lenstra, Phys. Rep. 416, 1 (2005).

[34] J. Ohtsubo, Semiconductor Lasers: Stability, Instability and Chaos, 3rd ed., Springer Series in Optical Sciences Vol. 111 (Springer, Berlin, 2013).

[35] T. B. Simpson and J. M. Liu, IEEE Photon. Technol. Lett. 9, 1322 (1997).

[36] E. K. Lau, X. Zhao, H.-K. Sung, D. Parekh, C. Chang-Hasnain, and M. C. Wu, Opt. Express 16, 6609 (2008). 